factors ${ }^{1}$. The aim of this study was to identify current practices within the UK, assess awareness of evidence-based GIM guidelines, and gauge opinion on the usefulness of such guidelines.

Methods An online survey composed of 10 questions was distributed via email to 984 recipients across the UK, including Gastroenterologists, Gastrointestinal surgeons and GPs with a specialist interest in Gastroenterology (GPwSI). Questions related to the clinical and endoscopic management of GIM, and the knowledge and value of GIM guidelines. Response data was analysed descriptively.

Results 75 responses were obtained with the majority from Consultant Gastroenterologists.

In an example case of an incidental finding of GIM in a 50 year old male with mild gastritis, a negative CLO test and no risk factors or alarm features suggestive of cancer: $51 \%$ would take no further action. $15 \%$ would test or empirically treat $\mathrm{H}$ pylori. 34\% would offer a repeat endoscopy, of which nearly half would offer at 1 year.

In clinical practice, $40 \%$ of respondents do not offer routine endoscopic surveillance for GIM. However, factors influencing the decision to offer surveillance include family history of gastric cancer (51\%); persistence of GIM at subsequent endoscopy $(19 \%)$ or patient request $(17 \%)$.

In the endoscopic surveillance for GIM: $45 \%$ take 3-5 biopsies and $42 \%$ take $6-10$ biopsies. The majority (53\%) do not use additional imaging techniques for example, narrow band imaging/chromoendoscopy. $62 \%$ do not routinely re-review GIM biopsies with a histopathologist.

If GIM is found, $29 \%$ inform patients of an increased gastric cancer risk, while $16 \%$ tell them there is no convincing evidence of increased risk. 31\% do not mention the diagnosis at all.

$96 \%$ are not aware of any guidelines on the management of GIM. 65\% think guidelines would be useful, 30\% do not feel strongly and only $5 \%$ would not find them useful.

Conclusion The management of GIM varies widely among clinicians in the UK. A large proportion do not offer endoscopic surveillance routinely, although consider alternative management strategies and take individual factors into account. In those that offer surveillance, the timing of repeat endoscopy varies, and there are differences in the endoscopic techniques employed. Although there are no existing universal UK guidelines, the majority of clinicians would welcome guidelines on the management of GIM.

Competing interests None.

Keywords gastric cancer, surveillance.

\title{
REFERENCE
}

1. Correa P, Piazuelo MB, Wilson KT. Pathology of gastric intestinal metaplasia: clinical implications. Am J Gastroenterol 2010;105:493-8.

\section{PTU-092 MANAGEMENT OF GASTRIC INTESTINAL METAPLASIA IN THE UK: A PRELIMINARY SURVEY}

doi:10.1136/gut.2011.239301.220

R Pandey, ${ }^{1, *}$ A Millar ${ }^{1}$ Department of Gastroenterology, North Middlesex University Hospital, London, UK

Introduction Gastric intestinal metaplasia (GIM) is considered a risk factor for gastric cancer ${ }^{1}$. Management and follow-up of these lesions is uncertain, as there are no standard UK guidelines. Current evidence suggests that patients with GIM should be considered for $\mathrm{H}$ pylori eradication and undergo endoscopic surveillance, depending on histological subtype and risk 